Research Article

\title{
Hyperstability of Some Functional Equations on Restricted Domain
}

\author{
Anna Bahyrycz and Jolanta Olko \\ Faculty of Applied Mathematics, AGH University of Science and Technology, Mickiewicza 30, 30-059 Kraków, Poland \\ Correspondence should be addressed to Anna Bahyrycz; bahyrycza@gmail.com
}

Received 20 April 2017; Accepted 30 May 2017; Published 16 July 2017

Academic Editor: Adrian Petrusel

Copyright (C) 2017 Anna Bahyrycz and Jolanta Olko. This is an open access article distributed under the Creative Commons Attribution License, which permits unrestricted use, distribution, and reproduction in any medium, provided the original work is properly cited.

The paper concerns functions which approximately satisfy, not necessarily on the whole linear space, a generalization of linear functional equation. A Hyers-Ulam stability result is proved and next applied to give conditions implying the hyperstability of the equation. The results may be used as tools in stability studies on restricted domains for various functional equations. We use the main theorem to obtain a few hyperstability results of Fréchet equation on restricted domain for different control functions.

\section{Introduction}

The famous Cauchy equation

$$
f(x+y)=f(x)+f(y)
$$

has a very long history, which started with A. M. Legendre in 1791 and C. F. Gauss in 1809, but the first important result about the solutions of (1) was proved by Cauchy (see [1]) and due to this outcome (1) is today named after him, as is mentioned in [2]. In the meantime, many authors have dealt with this equation, and next Fréchet in [3] studied an important generalization of Cauchy equation, characterizing the polynomials among the continuous mappings, namely, the equation

$$
\Delta_{h_{1} \cdots h_{n}}^{n} f(x)=0,
$$

where $\Delta$ denotes the difference operator defined as usual by

$$
\begin{aligned}
\Delta_{h} f(x) & =\Delta_{h}^{1} f(x):=f(x+h)-f(x), \\
\Delta_{h_{1} \cdots h_{n+1}}^{n+1} f(x) & =\Delta_{h_{n+1}}\left(\Delta_{h_{1} \cdots h_{n}}^{n} f(x)\right),
\end{aligned}
$$

for $x, h, h_{1}, \ldots, h_{n+1} \in \mathbb{R}, n \in \mathbb{N}$. From the result of Fréchet it follows that a continuous function $f: \mathbb{R} \rightarrow \mathbb{R}$ satisfies (2) for all $x$ and $h_{j}$ if and only if $f$ is a polynomial of degree smaller than or equal to $n-1$ (cf. $[2,4])$. As a generalization we can consider the functional equation

$$
\sum_{i=1}^{m} A_{i} g\left(\sum_{j=1}^{n} a_{i j} x_{j}\right)+A=0,
$$

where $g: X \rightarrow Y$ and $X, Y$ are the linear spaces over a field $\mathbb{F} \in\{\mathbb{R}, \mathbb{C}\}, A, a_{i j} \in \mathbb{F}$, and $A_{i} \in \mathbb{F} \backslash\{0\}, i \in\{1, \ldots, m\}, j \in$ $\{1, \ldots, n\}$.

The stability and hyperstability of the above functional equation and its particular cases were studied by many mathematicians (cf., e.g., [5-16]). Let us recall (see [9]) that the theory of Hyers-Ulam stability, in general, was motivated by a problem raised in 1940 by Ulam [17] and a partial answer to it was provided by Hyers [18]. The term hyperstability, in the meaning used here, was introduced presumably in [19] (cf. also [20]).

Our basic assumptions are the following. $X$ is a normed space over a field $\mathbb{F} \in\{\mathbb{R}, \mathbb{C}\}, E_{1}, E_{2}$ are the nonempty subsets of $X$ such that $E_{2} \subseteq E_{1} \subseteq X$, and $D$ is a nonempty subset of $\mathbb{F}$ such that $1 \in D, \mathbb{R}_{+}:=[0,+\infty)$. Denote $\mathbb{N}_{\leq k}:=\{1, \ldots, k\}$ and $\mathbb{N}_{k}:=\{l \in \mathbb{N}: l \geq k\}$, for $k \in \mathbb{N}$, and a sum of numbers over an empty set is defined to be zero. In the sequel, $\mathscr{P}(A)=$ $\{B \subset A: A \neq \emptyset\}$ for nonempty set $A$. 
We say that the equation

$$
\begin{aligned}
& \sum_{i=1}^{m} A_{i} g\left(\sum_{j=1}^{n} a_{i j} x_{j}\right)+A=0 \\
& x_{1}, \ldots, x_{n} \in E_{2}, \sum_{j=1}^{n} a_{1 j} x_{j}, \ldots, \sum_{j=1}^{n} a_{m j} x_{j} \in E_{1}
\end{aligned}
$$

is $\theta$-hyperstable in the class of functions $g: E_{1} \rightarrow Y$ (with a control function $\theta: E_{2}^{n} \rightarrow \mathbb{R}_{+}$), if $g: E_{1} \rightarrow Y$ satisfies the inequality

$$
\left\|\sum_{i=1}^{m} A_{i} g\left(\sum_{j=1}^{n} a_{i j} x_{j}\right)+A\right\| \leq \theta\left(x_{1}, \ldots, x_{n}\right),
$$

for all $x_{1}, \ldots, x_{n} \in E_{2}$, and $\sum_{j=1}^{n} a_{1 j} x_{j}, \ldots, \sum_{j=1}^{n} a_{m j} x_{j} \in E_{1}$ fulfills (5).

The above notion of hyperstability for functions defined on subsets of a linear space $X$ (not necessarily on the whole space but on the restricted domain) is a counterpart of hyperstability considered in [8]. In this paper we obtain, applying the fixed point theorem, some results concerning stability and hyperstability for (4) on restricted domain. Our outcomes allow studying the behavior of functions approximately fulfilling functional equations of this linear type on different subsets of the linear space, and this also applies to domain restrictions for control functions. This way we give a tool for the further Hyers-Ulam stability research. Its application is presented in the last section where some new as well as generalizations of known results on hyperstability of Cauchy and Fréchet equation are obtained.

\section{Auxiliary Result}

First we establish a counterpart of stability result from [7] on restricted domain, and to do this we use the fixed point theorem. This result will be needed to show our main theorem which provides criteria for hyperstability of many functional equations of the linear type on a restricted domain.

Lemma 1. Assume that $Y$ is a Banach space, $A=0$ or " $A \neq 0$ and $\sum_{i=1}^{m} A_{i} \neq 0$," and $\omega: D \rightarrow \mathbb{R}_{+}$. Suppose that there exist $\emptyset \neq I \subset \mathbb{N}_{\leq m}$ and $c_{1}, \ldots, c_{n} \in D$ such that

$$
\begin{aligned}
& \beta_{i}:=\sum_{j=1}^{n} a_{i j} c_{j} \in D, \\
& \quad c_{j} x \in E_{2}, \quad \beta_{i} x \in E_{2}, \quad i \in \mathbb{N}_{\leq m}, \quad j \in \mathbb{N}_{\leq n}, \quad x \in E_{2}, \\
& \beta_{l}=1, \quad l \in I, \\
& A_{I}:=\sum_{l \in I} A_{l} \neq 0, \\
& \gamma:=\sum_{i \notin I}\left|\frac{A_{i}}{A_{I}}\right| \omega\left(\beta_{i}\right)<1 .
\end{aligned}
$$

Let $\theta: E_{2}^{n} \rightarrow \mathbb{R}_{+}$satisfy the inequality

$$
\begin{aligned}
\theta\left(\beta_{i} x_{1}, \ldots, \beta_{i} x_{n}\right) \leq \omega\left(\beta_{i}\right) \theta & \left(x_{1}, \ldots, x_{n}\right), \\
& \quad i \in \mathbb{N}_{\leq m}, x_{1}, \ldots, x_{n} \in E_{2} .
\end{aligned}
$$

If $g: E_{1} \rightarrow Y$ fulfills the estimation

$$
\begin{aligned}
\left\|\sum_{i=1}^{m} A_{i} g\left(\sum_{j=1}^{n} a_{i j} x_{j}\right)+A\right\| \leq & \theta\left(x_{1}, \ldots, x_{n}\right), \\
& x_{1}, \ldots, x_{n} \in E_{2}, \sum_{j=1}^{n} a_{1 j} x_{j}, \ldots, \sum_{j=1}^{n} a_{m j} x_{j} \in E_{1},
\end{aligned}
$$

then there exists a unique function $G: E_{2} \rightarrow Y$ satisfying (5) such that

$$
\|g(x)-G(x)\| \leq \frac{\theta\left(c_{1} x, \ldots, c_{n} x\right)}{\left|A_{I}\right|(1-\gamma)}, \quad x \in E_{2} .
$$

Proof. Take $\emptyset \neq I \subset \mathbb{N}_{\leq m}$ and $c_{1}, \ldots, c_{n} \in D$ such that assumptions (7) and (8) hold.

First consider the case $A=0$. Taking $x \in E_{2}$ and substituting $x_{j}=c_{j} x, j \in \mathbb{N}_{\leq n}$, in (9) we have

$$
\left\|g(x)-\sum_{i \notin I} \frac{-A_{i}}{A_{I}} g\left(\beta_{i} x\right)\right\| \leq \frac{\theta\left(c_{1} x, \ldots, c_{n} x\right)}{\left|A_{I}\right|}, \quad x \in E_{2} .
$$

Define

$$
\begin{aligned}
(\mathscr{T} \xi)(x) & :=\sum_{i \notin I} \frac{-A_{i}}{A_{I}} \xi\left(\beta_{i} x\right), \quad \xi \in Y^{E_{2}}, x \in E_{2}, \\
(\Lambda \delta)(x) & :=\sum_{i \notin I}\left|\frac{-A_{i}}{A_{I}}\right| \delta\left(\beta_{i} x\right), \quad \delta \in \mathbb{R}_{+}^{E_{2}}, x \in E_{2}, \\
\varepsilon(x) & :=\frac{\theta\left(c_{1} x, \ldots, c_{n} x\right)}{\left|A_{I}\right|}, \quad x \in E_{2} .
\end{aligned}
$$

Then (11) may be rewritten as follows:

$$
\|g(x)-\mathscr{T} g(x)\| \leq \varepsilon(x), \quad x \in E_{2} .
$$

By induction, for every $x \in E_{2}$ and $k \in \mathbb{N}_{0}$

$$
\Lambda_{\varepsilon}^{k}(x) \leq \varepsilon(x) \gamma^{k} .
$$

Since $\gamma<1$, we see that

$$
\begin{aligned}
& \varepsilon^{*}(x):=\sum_{n=0}^{\infty}\left(\Lambda^{n} \varepsilon\right)(x) \leq \varepsilon(x) \sum_{n=0}^{\infty} \gamma^{n}=\frac{\varepsilon(x)}{1-\gamma}, \\
& x \in E_{2} .
\end{aligned}
$$

Notice that all assumptions of Theorem 1 in [21] are satisfied for operators (12) and (13) and $X=E_{2}$. According to this fixed point theorem, the function $G: E_{2} \rightarrow Y$ given by $G(x)=$ $\lim _{n \rightarrow \infty}\left(\mathscr{T}^{n} g\right)(x)$ for $x \in E_{2}$ is a fixed point of $\mathscr{T}$ fulfilling (10). 
It is sufficient to show that $G$ satisfies (5) (with $A=0$ ). First we prove that for every $k \in \mathbb{N} \cup\{0\}$ and every $x_{1}, \ldots, x_{n} \in$ $E_{2}$ such that $\sum_{j=1}^{n} a_{1 j} x_{j}, \ldots, \sum_{j=1}^{n} a_{m j} x_{j} \in E_{1}$

$$
\left\|\sum_{i=1}^{m} A_{i}\left(\mathscr{T}^{k} g\right)\left(\sum_{j=1}^{n} a_{i j} x_{j}\right)\right\| \leq \gamma^{k} \theta\left(x_{1}, \ldots, x_{n}\right) .
$$

Obviously, the case $k=0$ is just (9). Next, fix $k \in \mathbb{N} \cup\{0\}$, $x_{1}, \ldots, x_{n} \in E_{2}$, and $\sum_{j=1}^{n} a_{1 j} x_{j}, \ldots, \sum_{j=1}^{n} a_{m j} x_{j} \in E_{1}$ and assume that (18) holds. Then

$$
\begin{aligned}
\sum_{i=1}^{m} A_{i}\left(\mathscr{T}^{k+1} g\right)\left(\sum_{j=1}^{n} a_{i j} x_{j}\right) & \\
= & \sum_{i=1}^{m} A_{i} \mathscr{T}\left(\mathscr{T}^{k} g\right)\left(\sum_{j=1}^{n} a_{i j} x_{j}\right) \\
= & \sum_{i=1}^{m} A_{i} \sum_{p \notin I} \frac{-A_{p}}{A_{I}}\left(\mathscr{T}^{k} g\right)\left(\beta_{p} \sum_{j=1}^{n} a_{i j} x_{j}\right) \\
= & \sum_{p \notin I} \frac{-A_{p}}{A_{I}}\left[\sum_{i=1}^{m} A_{i}\left(\mathscr{T}^{k} g\right)\left(\sum_{j=1}^{n} a_{i j}\left(\beta_{p} x_{j}\right)\right)\right] .
\end{aligned}
$$

Thus by induction hypothesis and (8) we have

$$
\begin{aligned}
& \left\|\sum_{i=1}^{m} A_{i}\left(\mathscr{T}^{k+1} g\right)\left(\sum_{j=1}^{n} a_{i j} x_{j}\right)\right\| \\
& \leq \sum_{p \notin I}\left|\frac{-A_{p}}{A_{I}}\right| \gamma^{k} \theta\left(\beta_{p} x_{1}, \ldots, \beta_{p} x_{n}\right) \\
& \leq \sum_{p \notin I}\left|\frac{-A_{p}}{A_{I}}\right| \gamma^{k} \omega\left(\beta_{p}\right) \theta\left(x_{1}, \ldots, x_{n}\right) \\
& =\gamma^{k+1} \theta\left(x_{1}, \ldots, x_{n}\right),
\end{aligned}
$$

which finishes the proof of (18). Letting $k \rightarrow \infty$ in (18), we prove that $G$ satisfies (5), which completes the proof in the first case.

In the case where $A \neq 0$ and $\sum_{i=1}^{m} A_{i} \neq 0$, the function $h(x):=g(x)+A / \sum_{i=1}^{m} A_{i}, x \in E_{1}$, satisfies

$$
\left\|\sum_{i=1}^{m} A_{i} h\left(\sum_{j=1}^{n} a_{i j} x_{j}\right)\right\| \leq \theta\left(x_{1}, \ldots, x_{n}\right),
$$

for $x_{1}, \ldots, x_{n} \in E_{2}$, and $\sum_{j=1}^{n} a_{1 j} x_{j}, \ldots, \sum_{j=1}^{n} a_{m j} x_{j} \in E_{1}$. Consequently, we conclude that there exists a unique function $H: E_{2} \rightarrow Y$ such that

$$
\|h(x)-H(x)\| \leq \frac{\theta\left(c_{1} x, \ldots, c_{n} x\right)}{\left|A_{I}\right|(1-\gamma)}, \quad x \in E_{2} .
$$

Obviously $G(x):=H(x)-A / \sum_{i=1}^{m} A_{i}$ and $x \in E_{2}$ is the desired function.
Denote

$$
\begin{gathered}
X_{0}:=X \backslash\{0\}, \\
\mathbb{F}_{0}:=\mathbb{F} \backslash\{0\} .
\end{gathered}
$$

Remark 2. It is easily seen that functions

(i) $\theta_{1}\left(x_{1}, \ldots, x_{n}\right)=C$;

(ii) $\theta_{2}\left(x_{1}, \ldots, x_{n}\right)=C \sum_{j=1}^{n}\left\|c_{j} x_{j}\right\|^{k_{j}}$;

(iii) $\theta_{3}\left(x_{1}, \ldots, x_{n}\right)=C \max \left\{\left\|c_{j} x_{j}\right\|^{k_{j}}: j \in\{1, \ldots, n\}\right\}$;

(iv) $\theta_{4}\left(x_{1}, \ldots, x_{n}\right)=C \Pi_{j=1}^{n}\left\|x_{j}\right\|^{k_{j}}$;

(v) $\theta_{5}\left(x_{1}, \ldots, x_{n}\right)=C \Pi_{j=1}^{n}\left\|x_{j}\right\|^{t_{j}}+S \sum_{j=1}^{n}\left\|c_{j} x_{j}\right\|^{k_{j}}$;

with some $C, S \in[0,+\infty), C+S>0, c_{j} \in \mathbb{F}_{0}$, and $k_{j}, t_{j} \neq 0$ fulfill condition (8) for some function $\omega$. For instance, in case (iv) we can use $\omega_{4}(\beta)=|\beta|^{\sum_{j=1}^{n} k_{j}}$ for $\beta \in D$, and in case (v) we may take

$$
\omega_{5}(\beta):= \begin{cases}|\beta|^{\max \left\{k_{1}, \ldots, k_{n}, t\right\}} & \text { for }|\beta| \geq 1 \\ |\beta|^{\min \left\{k_{1}, \ldots, k_{n}, t\right\}} & \text { for }|\beta|<1,\end{cases}
$$

where $t=\sum_{j=1}^{n} t_{j}$. If there exists at least one $j_{0} \in \mathbb{N}_{\leq n}$ such that $k_{\mathrm{j}_{0}}<0$ or $t_{j_{0}}<0$ then $0 \notin D$.

\section{The Main Result}

We can now state a counterpart (on restricted domain) of Theorem 2.1 in [8], concerning $\theta$-hyperstability of (5).

Theorem 3. Let $Y$ be a normed space over $\mathbb{F}, A=0$ or " $A \neq$ 0 and $\sum_{i=1}^{m} A_{i} \neq 0, " g: E_{1} \rightarrow Y, \omega: D \rightarrow \mathbb{R}_{+}$, and $\theta$ : $E_{2}^{n} \rightarrow \mathbb{R}_{+}$satisfy (9). If there exist $\emptyset \neq I \subset \mathbb{N}_{\leq m}$ and a sequence $\left\{\left(c_{1}^{k}, \ldots, c_{n}^{k}\right)\right\}_{k \in \mathbb{N}}$ of elements of $D^{n}$ such that

$$
\begin{aligned}
\beta_{i}^{k} & :=\sum_{j=1}^{n} a_{i j} c_{j}^{k} \in D, \\
c_{j}^{k} x & \in E_{2}, \\
\beta_{i}^{k} x & \in E_{2}, \\
i & \in \mathbb{N}_{\leq m}, \quad j \in \mathbb{N}_{\leq n}, \quad k \in \mathbb{N}, x \in E_{2}, \\
\beta_{l}^{k} & =1, \quad l \in I, \\
A_{I} & :=\sum_{l \in I} A_{l} \neq 0,
\end{aligned}
$$

$$
\begin{aligned}
\lim _{k \rightarrow \infty} \sum_{i \notin I}\left|\frac{A_{i}}{A_{I}}\right| \omega\left(\beta_{i}^{k}\right) & <1, \\
\theta\left(\beta_{i}^{k} x_{1}, \ldots, \beta_{i}^{k} x_{n}\right) & \leq \omega\left(\beta_{i}^{k}\right) \theta\left(x_{1}, \ldots, x_{n}\right) \\
i & \in \mathbb{N}_{\leq m}, k \in \mathbb{N}, x_{1}, \ldots, x_{n} \in E_{2}, \\
\lim _{k \rightarrow \infty} \theta\left(c_{1}^{k} x, \ldots, c_{n}^{k} x\right) & =0,
\end{aligned}
$$

then $g$ satisfies (5). 
Proof. We can certainly assume that $Y$ is a Banach space, for if not we replace it by its completion.

Take $\emptyset \neq I \subset \mathbb{N}_{\leq m}$ and the sequence $\left\{\left(c_{1}^{k}, \ldots, c_{n}^{k}\right)\right\}_{k \in \mathbb{N}}$ of the elements of $D^{n}$ such that conditions (25), (26), and (27) hold. Hence we can find $\varepsilon \in(0,1)$ and $k_{0} \in \mathbb{N}$ such that

$$
\gamma_{k}:=\sum_{i \notin I}\left|\frac{A_{i}}{A_{I}}\right| \omega\left(\beta_{i}^{k}\right)<1-\varepsilon, \quad k \geq k_{0} .
$$

Therefore, according to Lemma 1 , for each $k \in \mathbb{N}_{k_{0}}$ there exists a unique function $G_{k}: E_{2} \rightarrow Y$ satisfying equation (5) such that

$$
\left\|g(x)-G_{k}(x)\right\| \leq \frac{\theta\left(c_{1}^{k} x, \ldots, c_{n}^{k} x\right)}{\left|A_{I}\right|\left(1-\gamma_{k}\right)}, \quad x \in E_{2} .
$$

In this way we obtain the sequence $\left\{G_{k}\right\}_{k \in \mathbb{N}_{k_{0}}}$ of functions satisfying (5). Letting in (30) $k \rightarrow \infty$, we get, using (28), that $g$ satisfies (5).

\section{Applications}

Using the above outcome we can get the results for the particular form of (5), as, for instance, for Fréchet functional equation, presented in this section.

Corollary 4. Assume that $Y$ is a normed space over $\mathbb{F}, n \in \mathbb{N}_{2}$, $E$ is a nonempty subset of $X_{0}$ such that there exists a positive integer $l_{0}$ with

$$
-x, l x \in E, \quad x \in E, l \in \mathbb{N}_{l_{0}} .
$$

If $g: E \rightarrow Y$ fulfills the inequality

$$
\begin{aligned}
\left\|\Delta_{x_{2} \cdots x_{n}}^{n-1} g\left(x_{1}\right)\right\| & \leq C \Pi_{j=1}^{n}\left\|x_{j}\right\|^{k_{j}}, \\
\sum_{j \in T} x_{j} & \in E, \quad T \in \mathscr{P}\left(\mathbb{N}_{\leq n}\right),
\end{aligned}
$$

with $C>0$ and $\sum_{j=1}^{n} k_{j}<0$, then $g$ is a solution of the equation

$$
\begin{aligned}
\Delta_{x_{2} \cdots x_{n}}^{n-1} g\left(x_{1}\right) & =0, \\
\sum_{j \in T} x_{j} & \in E, \quad T \in \mathscr{P}\left(\mathbb{N}_{\leq n}\right) .
\end{aligned}
$$

Proof. Put $E:=E_{2}=E_{1}, D:=\mathbb{Z}_{0}$, and $\omega(\beta):=|\beta|^{\sum_{j=1}^{n} k_{j}}$ for $\beta \in \mathbb{Z}_{0}$ and consider two cases:

(a) $\sum_{j=2}^{n} k_{j}<0$

(b) $\sum_{j=2}^{n} k_{j} \geq 0$.

In the case (a) we define the sequence $\left\{\left(c_{1}^{k}, \ldots, c_{n}^{k}\right)\right\}_{k \in \mathbb{N}}$ as follows:

$$
c_{j}^{k}:= \begin{cases}k+l_{0} & \text { for } j \neq 1 \\ 1 & \text { for } j=1\end{cases}
$$

It follows easily that the assumptions of Theorem 3 are satisfied with $m=2^{n}$ and $I=\left\{2^{n}\right\}$.

In case (b) we can find $j_{0} \in\{2, \ldots, n\}$ such that $k_{j_{0}} \geq 0$. Define

$$
c_{j}^{k}:= \begin{cases}k+l_{0} & \text { for } j \neq j_{0} \\ 1-k-l_{0} & \text { for } j=j_{0} .\end{cases}
$$

There exists $i_{0} \in\left\{1, \ldots, 2^{n}\right\}$ such that $a_{i_{0} 1}=a_{i_{0} j_{0}}=1$ and $a_{i_{0} j}=0$ for $j \in\{2, \ldots, n\} \backslash\left\{1, j_{0}\right\}$. We will verify that we can apply Theorem 3 for $I=\left\{i_{0}\right\}$. Namely,

$$
\begin{aligned}
& \sum_{j=1}^{n} a_{i_{0} j} c_{j}^{k}=k+l_{0}+1-k-l_{0}=1, \\
& k+l_{0} \leq \sum_{j=1}^{n} a_{i j} c_{j}^{k} \leq\left(k+l_{0}\right)(n-1) \quad \text { for } i \neq i_{0} .
\end{aligned}
$$

Therefore

$$
\begin{aligned}
& \lim _{k \rightarrow \infty} \sum_{i \neq i_{0}}\left|\sum_{j=1}^{n} a_{i j} c_{j}^{k}\right|^{\sum_{j=1}^{n} k_{j}}=0, \\
& \lim _{k \rightarrow \infty} C \Pi_{j=1}^{n}\left\|c_{j}^{k} x\right\|^{k_{j}} \\
& \quad=\lim _{k \rightarrow \infty}\left|k+l_{0}\right|^{\sum_{j \neq j_{0}} k_{j}}\left|k+l_{0}-1\right|^{k_{j_{0}}}\|x\|^{\sum_{j=1}^{n} k_{j}} \\
& \quad \leq \lim _{k \rightarrow \infty}\left|k+l_{0}\right|^{\sum_{j=1}^{n} k_{j}}\|x\|^{\sum_{j=1}^{n} k_{j}}=0 .
\end{aligned}
$$

Thus conditions (26) and (28) hold, which finishes the proof.

Corollary 5. Assume that $Y$ is a normed space over $\mathbb{F}, n \in$ $\mathbb{N}_{2}$, and $E$ is a nonempty subset of $X_{0}$ such that there exists a positive integer $l_{0}$ with (31).

If $g: E \rightarrow Y$ fulfills the inequality

$$
\begin{aligned}
\left\|\Delta_{x_{2} \cdots x_{n}}^{n-1} g\left(x_{1}\right)\right\| \leq & \left(\sum_{j=1}^{n} C_{j}\left\|x_{j}\right\|^{k_{j}}\right)^{w}, \\
& \sum_{j \in T} x_{j} \in E, T \in \mathscr{P}\left(\mathbb{N}_{\leq n}\right),
\end{aligned}
$$

with $C_{j}>0$ and $w k_{j}<0$, for $j \in\{1, \ldots, n\}$, then $g$ is a solution of (33).

Proof. Putting $E:=E_{2}=E_{1}, D:=\mathbb{Z}_{0}$, and $\omega(\beta):=|\beta|^{-v|w|}$ for $\beta \in \mathbb{Z}_{0}$, where $v:=\min \left\{\left|k_{1}\right|, \ldots,\left|k_{n}\right|\right\}, I:=\{1\}$, and

$$
c_{j}^{k}:= \begin{cases}(n-1)\left(k+l_{0}\right)+1 & \text { for } j=1 \\ -k-l_{0} & \text { for } j>1,\end{cases}
$$

we get that

$$
\begin{gathered}
\beta_{1}^{k}=1, \\
k+l_{0}+1 \leq \beta_{i}^{k} \leq(n-1)\left(k+l_{0}\right)+1 \text { for } i>1,
\end{gathered}
$$


and therefore $\lim _{k \rightarrow \infty}\left|\beta_{i}^{k}\right|=+\infty$ for $i \neq 1$, and consequently condition (26) is satisfied. Also, condition (28) holds, because

$$
\begin{gathered}
\lim _{k \rightarrow \infty}\left(C_{1}\left(\left|(n-1)\left(k+l_{0}\right)+1\right|\|x\|\right)^{k_{1}}\right. \\
\left.+\sum_{j=2}^{n} C_{j}\left(\left|k+l_{0}\right|\|x\|\right)^{k_{j}}\right)^{w}=0,
\end{gathered}
$$

which with Theorem 3 completes the proof.

Denote

$$
\begin{aligned}
F\left(f, x_{1}, \ldots, x_{n}\right)=\Delta_{x_{1} \cdots x_{n}}^{n} f(0)+(-1)^{n+1} f(0), & \\
& f \in Y^{X}, x_{1}, \ldots, x_{n} \in X .
\end{aligned}
$$

Corollary 6. Assume that $Y$ is a normed space over $\mathbb{F}, n \in$ $\mathbb{N}_{2}$, and $E$ is a nonempty subset of $X_{0}$ such that there exists a positive integer $l_{0}$ with

$$
l x \in E, \quad x \in E, l \in \mathbb{N}_{l_{0}} .
$$

If $g: E \rightarrow Y$ fulfills the inequality

$$
\begin{aligned}
\left\|F\left(g, x_{1}, \ldots, x_{n}\right)\right\| \leq C \Pi_{j=1}^{n}\left\|x_{j}\right\|^{k_{j}}, \\
\qquad \sum_{j \in T} x_{j} \in E, T \in \mathscr{P}\left(\mathbb{N}_{\leq n}\right),
\end{aligned}
$$

with $C>0$ and $\sum_{j=1}^{n} k_{j}<0$, then $g$ is a solution of the equation

$$
F\left(g, x_{1}, \ldots, x_{n}\right)=0, \quad \sum_{j \in T} x_{j} \in E, T \in \mathscr{P}\left(\mathbb{N}_{\leq n}\right) .
$$

Proof. Put $E:=E_{2}=E_{1}, D:=\mathbb{Z}_{0}$, and $\omega(\beta):=|\beta|^{\sum_{j=1}^{n} k_{j}}$ for $\beta \in \mathbb{Z}_{0}$. There exist $j_{0} \in\{1, \ldots, n\}$ and $i_{0} \in\left\{1, \ldots, 2^{n}-1\right\}$, such that $\sum_{j \neq j_{0}}^{n} k_{j}<0, a_{i_{0} j_{0}}=1$, and $a_{i_{0} j}=0$ for $j \neq j_{0}$. Define the sequence $\left\{\left(c_{1}^{k}, \ldots, c_{n}^{k}\right)\right\}_{k \in \mathbb{N}}$ as follows:

$$
c_{j}^{k}:= \begin{cases}k+l_{0} & \text { for } j \neq j_{0} \\ 1 & \text { for } j=j_{0} .\end{cases}
$$

The assumptions of Theorem 3 are fulfilled with $m=2^{n}-1$ and $I=\left\{i_{0}\right\}$, as is easy to check, which finishes the proof.

In the same way as Corollary 5 we can prove the following corollary.

Corollary 7. Assume that $Y$ is a normed space over $\mathbb{F}, n \in$ $\mathbb{N}_{2}$, and $E$ is a nonempty subset of $X_{0}$ such that there exists a positive integer $l_{0}$ with (31).

If $g: E \rightarrow Y$ fulfills the inequality

$$
\begin{array}{r}
\left\|F\left(g, x_{1}, \ldots, x_{n}\right)\right\| \leq\left(\sum_{j=1}^{n} C_{j}\left\|x_{j}\right\|^{k_{j}}\right)^{w}, \\
\sum_{j \in T} x_{j} \in E, \quad T \in \mathscr{P}\left(\mathbb{N}_{\leq n}\right)
\end{array}
$$

with $C_{j}>0$ and $w k_{j}<0$ for $j \in\{1, \ldots, n\}$, then $g$ is a solution of (45).
Remark 8. Observe that putting in the above two corollaries $n=2$ we get hyperstability results for Cauchy equation. Corollary 7 generalizes the result from [9] (see Theorem 1.2), where $n=2$ and $k_{1}=k_{2}<0$ (in our considerations $k_{1}, k_{2}<0$ may be different). Corollaries 6 and 7 generalize the outcomes from [6].

We end the paper with an example, which shows that the additional assumption on $E$, appearing in the above corollaries, is essential.

Example 9. Let $X=Y=\mathbb{R}, E=[-1,0) \cup(0,1]$, and $f: E \rightarrow$ $\mathbb{R}$ be defined by $f(x)=\sin x / 2^{n}, x \in E$. Then $f$ satisfies the estimations

$$
\begin{aligned}
\left\|\Delta_{x_{2} \cdots x_{n}}^{n-1} f\left(x_{1}\right)\right\| & <1 \leq \Pi_{j=1}^{n}\left\|x_{j}\right\|^{-1}, \\
\left\|F\left(f, x_{1}, \ldots, x_{n}\right)\right\| & <1 \leq \Pi_{j=1}^{n}\left\|x_{j}\right\|^{-1}, \\
\left\|\Delta_{x_{2} \cdots x_{n}}^{n-1} f\left(x_{1}\right)\right\| & <1 \leq \sum_{j=1}^{n}\left\|x_{j}\right\|^{-1}, \\
\left\|F\left(f, x_{1}, \ldots, x_{n}\right)\right\| & <1 \leq \sum_{j=1}^{n}\left\|x_{j}\right\|^{-1},
\end{aligned}
$$

for $\sum_{j \in T} x_{j} \in E$, and $T \in \mathscr{P}\left(\mathbb{N}_{\leq n}\right)$ but $f$ satisfies neither (33) nor (45).

\section{Conflicts of Interest}

The authors declare that there are no conflicts of interest regarding the publication of this paper.

\section{References}

[1] A. L. Cauchy, "Cours d'analyse de l'École royale polytechnique," Paris, 1821.

[2] J. M. Almira and A. J. López-Moreno, "On solutions of the Fréchet functional equation," Journal of Mathematical Analysis and Applications, vol. 332, no. 2, pp. 1119-1133, 2007.

[3] M. Fréchet, "Un définition fonctionnelle des polynômes," Nouvelles Annales de Mathematiques, vol. 9, no. 4, pp. 145-162, 1909.

[4] M. E. Gordji, H. Khodaei, and T. M. Rassias, "A functional equation having monomials and its stability," in Handbook of Functional Equations, vol. 96, pp. 181-197, Springer, New York, NY, USA, 2014.

[5] A. Bahyrycz and M. Piszczek, "Hyperstability of the Jensen functional equation," Acta Mathematica Hungarica, vol. 142, no. 2, pp. 353-365, 2014.

[6] A. Bahyrycz, J. Brzdęk, M. Piszczek, and J. Sikorska, "Hyperstability of the Fréchet equation and a characterization of inner product spaces," Journal of Function Spaces and Applications, vol. 2013, Article ID 496361, 2013.

[7] A. Bahyrycz and J. Olko, "On stability of the general linear equation," Aequationes Mathematicae, vol. 89, no. 6, pp. 14611474, 2015.

[8] A. Bahyrycz and J. Olko, "Hyperstability of general linear functional equation," Aequationes Mathematicae, vol. 90, no. 3, pp. 527-540, 2016. 
[9] J. Brzdęk, "Hyperstability of the Cauchy equation on restricted domains," Acta Mathematica Hungarica, vol. 141, no. 1-2, pp. 5867, 2013.

[10] J. Brzdęk and K. Ciepliński, "Hyperstability and superstability," Abstract and Applied Analysis, vol. 2013, Article ID 401756, 13 pages, 2013.

[11] L. Cădariu and V. Radu, "Fixed points and the stability of Jensen's functional equation," JIPAM. Journal of Inequalities in Pure and Applied Mathematics, vol. 4, no. 1, Article 4, 7 pages, 2003.

[12] D. Popa and I. Raşa, "The Fréchet functional equation with application to the stability of certain operators," Journal of Approximation Theory, vol. 164, no. 1, pp. 138-144, 2012.

[13] M. Piszczek, "Remark on hyperstability of the general linear equation," Aequationes Mathematicae, vol. 88, no. 1-2, pp. 163$168,2014$.

[14] M. Piszczek and J. Szczawińska, "Hyperstability of the drygas functional equation," Journal of Function Spaces and Applications, vol. 2013, Article ID 912718, 4 pages, 2013.

[15] F. Skof, "On the stability of functional equations on a restricted domain and a related topic," in Stability of mappings of HyersUlam type, Th. M. Rassias and J. Tabor, Eds., pp. 141-151, Hadronic Press, Florida, Fla, USA, 1994.

[16] D. Zhang, "On hyperstability of generalised linear functional equations in several variables," Bulletin of the Australian Mathematical Society, vol. 92, no. 2, pp. 259-267, 2015.

[17] S. M. Ulam, Problems in Modern Mathematics, Science Editions, Chapter 6, Wiley, New York, NY, USA, 1960.

[18] D. H. Hyers, "On the stability of the linear functional equation," Proceedings of the National Academy of Sciences of the United States of America, vol. 27, pp. 222-224, 1941.

[19] G. Y. Maksa and Z. S. Páles, "Hyperstability of a class of linear functional equations," Acta Mathematica Academiae Paedagogicae Nyiregyhaziensis, vol. 17, pp. 107-112, 2001.

[20] G. Maksa, "The stability of the entropy of degree alpha," Journal of Mathematical Analysis and Applications, vol. 346, no. 1, pp. 17-21, 2008.

[21] J. Brzdęk, J. Chudziak, and Z. Páles, "A fixed point approach to stability of functional equations," Nonlinear Analysis: Theory, Methods \& Applications, vol. 74, no. 17, pp. 6728-6732, 2011. 


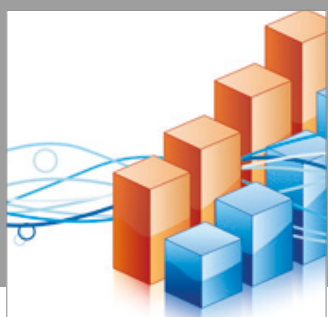

Advances in

Operations Research

vatersals

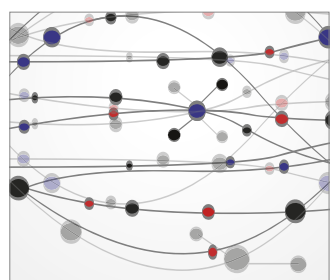

\section{The Scientific} World Journal
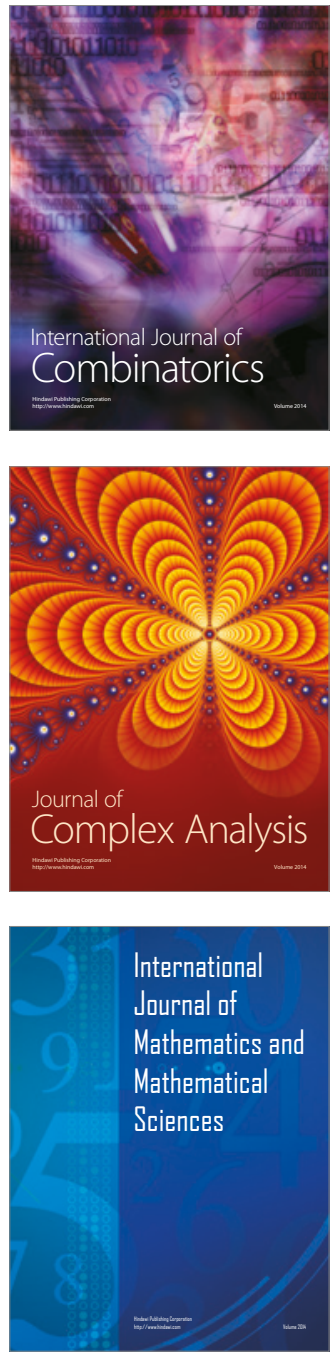
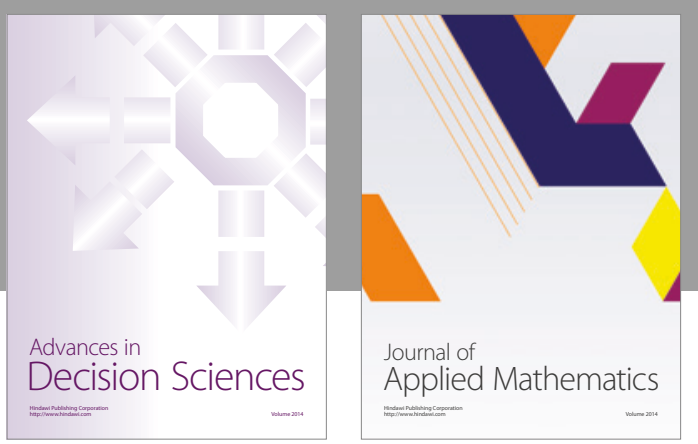

Algebra

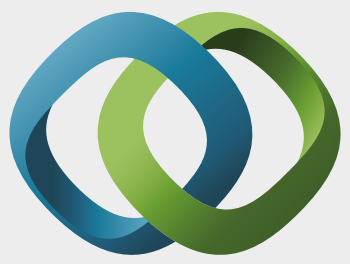

\section{Hindawi}

Submit your manuscripts at

https://www.hindawi.com
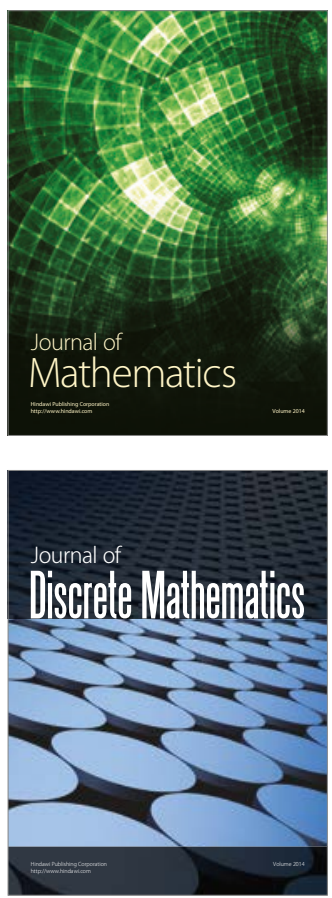

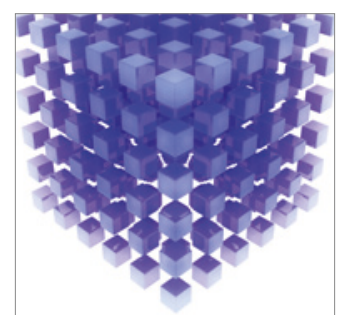

Mathematical Problems in Engineering
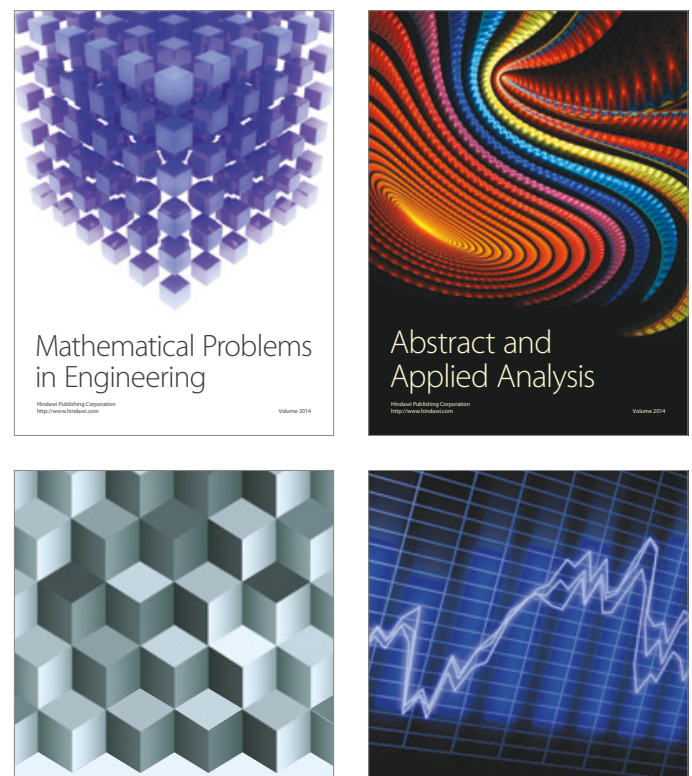

Journal of

Function Spaces

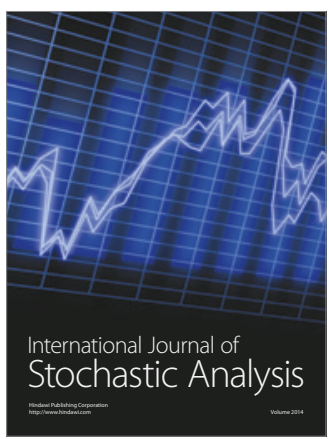

Probability and Statistics
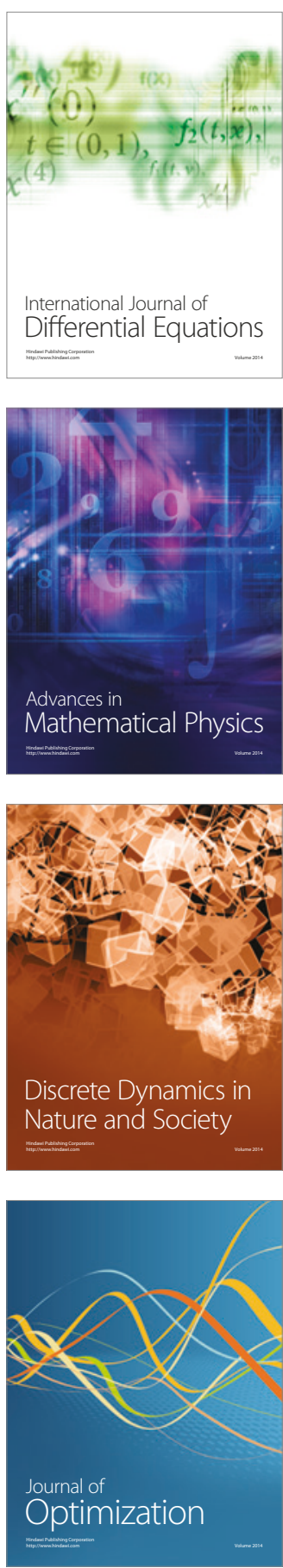